\title{
Adenovirus, calicivirus and astrovirus detection in fecal samples of hospitalized children with acute gastroenteritis from Campo Grande, MS, Brazil
}

\author{
Marcia Sueli Assis Andreasi $/{ }^{+}$, Divina das Dores de Paula Cardoso', Sonia Maria Fernandes, \\ Ines Aparecida Tozetti, Ana Maria Tavares Borges', Fabíola Souza Fiaccadori', \\ Rodrigo Alessandro Tôgo Santos' ${ }^{1}$, Menira Souza ${ }^{1}$
}

Departamento de Patologia, Centro de Ciências Biológicas e da Saúde, Universidade Federal de Mato Grosso do Sul, Cidade Universitária, 79070-900 Campo Grande, MS, Brasil 'Instituto de Patologia Tropical e Saúde Pública, Universidade Federal de Goiás, Goiânia, GO, Brasil

We analyzed fecal samples from hospitalized children up to three years of age with acute gastroenteritis at Campo Grande, Mato Grosso do Sul, Brazil, from May 2000-January 2004. Astrovirus and calicivirus were detected by Reverse Transcription-Polymerase Chain Reaction and adenovirus was detected using the Rotavirus and Adenovirus combined immunoenzyme assay. Astrovirus, adenovirus and calicivirus were detected at rates of $3.1 \%$, $3.6 \%$ and $7.6 \%$, respectively. These results re-emphasize the need for the establishment of regional vigilance systems to evaluate the impact of enteric viruses on viral gastroenteritis.

Key words: calicivirus - adenovirus - astrovirus - gastroenteritis

Acute gastroenteritis is one of the most common childhood diseases, especially in developing countries. Annually, 2.5 million deaths are estimated to occur, which greatly impact the population of children younger than five years of age (Girard et al. 2006). Over 20 different types of viruses have been identified as etiological agents for this disease (Wilhelmi et al. 2003). Group A rotavirus are considered to be the main agents, followed by calicivirus, adenovirus and astrovirus. Globally, these viruses are responsible for diarrhea episodes in hospitalized children, with detection rates varying from $20-60 \%$, $3.5-29.3 \%, 1-31 \%$ and $1.8-16 \%$, respectively (Jakab et al. 2005, Caracciolo et al. 2007, Fodha et al. 2007). In Brazil, similar detection rates have been observed (Resque et al. 2007, Soares et al. 2007), including those studied in Goiânia, located in the Central-West region of Brazil (Cardoso et al. 2002, Santos et al. 2007).

In Campo Grande, the unique data concerning the etiology of acute gastroenteritis in children refer to group A rotavirus, with detection rates of $23.2 \%$ detected in children up to three years old (Andreasi et al. 2007). Considering the need for constant monitoring of viral gastroenteritis in establishing prevention programs, the present study aimed to detect enteric viruses in fecal samples obtained from hospitalized children with acute diarrhea in Campo Grande.

Financial support: $\mathrm{CNPq}$

+ Corresponding author:msaa@nin.ufms.br

Received 3 June 2008

Accepted 16 September 2008

\section{PATIENTS, MATERIAL AND METHODS}

This study was performed between May 2000-January 2004, using fecal samples collected from hospitalized children, under three years of age, with acute gastroenteritis at Santa Casa de Misericórdia from Campo Grande and Hospital Universitário da Universidade Federal de Mato Grosso do Sul. The inclusion criteria were defined as the occurrence of acute gastroenteritis, characterized as liquid or semi liquid, with three or more evacuations in a $24 \mathrm{~h}$ period. The collection of fecal samples was carried out in the first $24 \mathrm{~h}$ of hospitalization, after written informed consent was provided by parents or legal guardians. This study was approved by the Ethical Committee on Research of the Universidade Federal de Mato Grosso do Sul (protocol 599).

Astrovirus and calicivirus were detected by Reverse Transcription-Polymerase Chain Reaction (RT-PCR), using $20 \%$ fecal suspension (phosphate buffered saline $\mathrm{pH}$ 7.4). Viral ssRNA extraction was performed according to the Boom et al. (1990) method modified by Cardoso et al. (2002). We used the random primer pd(N)6 (Random Hexamer, Amersham Biosciences) to obtain cDNA and the PCR was carried out using the primers Mon 269 and 270 for astrovirus (Noel et al. 1995) and Ni/E3 (Green et al. 1995), JV12/13 (Vinjé et al. 1997) and 289/290 (Jiang et al. 1999) for calicivirus. Samples known to be positive for astrovirus and calicivirus and sterile Milli-Q water were included in all reactions as positive and negative controls, respectively. Adenovirus was detected by Rotavirus and Adenovirus Combined Immunoenzyme Assay, following methodology described by Pereira et al. (1985) to detect the Mastadenovirus genus. Statistical analysis was carried out using Epi-info software, version 6.0. Positivity proportion analysis was done by Chi-square test $\left(\chi^{2}\right)$ with a $95 \%$ confidence interval and Fisher's exact test, when necessary. 


\section{RESULTS}

Adenovirus and calicivirus were examined in 415 and 406 fecal samples, respectively, while 354 fecal samples were used for astrovirus detection. The positivity rates observed were of $3.1 \%, 3.6 \%$ and $7.6 \%$ for astrovirus, adenovirus and calicivirus, respectively. The three types of viruses were detected only in children less than 24 months old, although no statistically significant differences were observed regarding positivity for any virus researched among any of the age groups (Table I).

There was no statistically significant difference in viral positivity between children with diarrhea alone, as compared to children exhibiting additional symptoms, such as vomiting, fever and abdominal pain. However, of the entire group of 316 children whose samples were used for detection of all three types of viruses, and for which there was complete information regarding clinical status, 85 also presented with respiratory complaints. Analysis of viral positivity with respect to the presence or absence of respiratory complaints demonstrated that astrovirus was detected only in children without respiratory complaints, whereas adenovirus and calicivirus were detected in three and seven children with respiratory symptoms, respectively.

Ten children demonstrated positivity to more than one type of virus. Among these, nine were infected with group A rotavirus; five out of these nine children were co-infected with astrovirus, two with adenovirus and two with calicivirus. The other child showed positivity to astrovirus and calicivirus. The results relative to group A rotavirus have been published previously (Andreasi 2007).

Analysis of viral detection with respect to the number of days of diarrhea was performed in 308 children. The highest detection rate of astrovirus was observed in the first day of diarrhea (14.2\%). Adenovirus was mainly detected in children on the third day of disease $(6.6 \%)$, while calicivirus showed increasing positivity related to days of disease, with the highest detection rates beginning on the fifty day of disease $(8.8 \%-12.1 \%$; $p=0.034)$. Calicivirus was mainly detected in female children.

All three virus types were predominantly detected in 2002 and 2003, with calicivirus being predominant in 2002 (Table II). Additionally, all types of viruses circulated in all months of the year, and, although calicivirus showed high occurrence in March-April, this difference was not observed when analysis was performed with respect to the different seasons of the year.

From the 31 positive samples for calicivirus, 20 showed positivity to the $\mathrm{Ni} / \mathrm{E} 3$ primers, eight to the $289 / 290$ primers, two to the JV12/13 primers and one to the $\mathrm{Ni} / \mathrm{E} 3$ and $289 / 290$ primers.

There was no information regarding bacterial or parasite infection among the children in this study. We did not observe any correlation between viral infection and duration of symptoms or days of hospitalization.

TABLE I

Distribution of the astrovirus, adenovirus and calicivirus positive samples related to age groups

\begin{tabular}{|c|c|c|c|c|c|c|}
\hline \multirow[b]{2}{*}{ Age group (months) } & \multicolumn{2}{|c|}{ Astrovirus } & \multicolumn{2}{|c|}{ Adenovirus } & \multicolumn{2}{|c|}{ Calicivirus } \\
\hline & Positive/total & $\%$ & Positive/total & $\%$ & Positive/total & $\%$ \\
\hline$<1$ & $-/ 1$ & - & $-/ 2$ & - & $-/ 2$ & - \\
\hline $1-6$ & $4 / 115$ & 3.5 & $4 / 130$ & 3.0 & $10 / 126$ & 7.9 \\
\hline $7-12$ & $5 / 139$ & 3.5 & $5 / 168$ & 3.0 & $15 / 163$ & 9.2 \\
\hline $13-24$ & $2 / 90$ & 2.2 & $6 / 105$ & 5.7 & $6 / 104$ & 5.8 \\
\hline $25-36$ & $-/ 8$ & - & $-/ 9$ & - & $-/ 10$ & - \\
\hline $37-48$ & $-/ 1$ & - & $-/ 1$ & - & $-/ 1$ & - \\
\hline Total & $11 / 354$ & 3.1 & $15 / 415$ & 3.6 & $31 / 406$ & 7.6 \\
\hline $\mathrm{p}$ & \multicolumn{2}{|c|}{$0.6970^{a}$} & \multicolumn{2}{|c|}{$0.3485^{a}$} & \multicolumn{2}{|c|}{$0.5211^{b}$} \\
\hline
\end{tabular}

$a$ : Fisher's exact test; $b: \chi^{2}=0.41$.

TABLE II

Distribution of astrovirus, adenovirus and calicivirus positive samples related to years of collection, 2000/2004

\begin{tabular}{|c|c|c|c|c|c|c|}
\hline \multirow[b]{2}{*}{ Year } & \multicolumn{2}{|c|}{ Astrovirus } & \multicolumn{2}{|c|}{ Adenovirus } & \multicolumn{2}{|c|}{ Calicivirus } \\
\hline & Positive/total & $\%$ & Positive/total & $\%$ & Positive/total & $\%$ \\
\hline 2000 & $1 / 15$ & 6.7 & $-/ 15$ & & $-/ 15$ & \\
\hline 2001 & $-/ 12$ & & $-/ 12$ & & $-/ 12$ & \\
\hline 2002 & $3 / 63$ & 4.8 & $1 / 65$ & 1.5 & $12 / 67$ & 17.9 \\
\hline 2003 & $7 / 244$ & 2.9 & $14 / 298$ & 4.7 & $19 / 286$ & 6.6 \\
\hline 2004 & $-/ 20$ & & $-/ 25$ & & $-/ 26$ & \\
\hline $\mathrm{p}$ & \multicolumn{2}{|c|}{$0.4349^{a}$} & \multicolumn{2}{|c|}{$0.4876^{a}$} & \multicolumn{2}{|c|}{$0.0034^{b}$} \\
\hline
\end{tabular}


We performed genotypic characterization on the astrovirus samples and found that six samples were HAstV-1, three were HAstV-2 and one was HAstV-4. Co-infection was observed in 10 samples, with five samples testing positive for rotavirus and astrovirus, four samples for rotavirus and adenovirus and one sample for astrovirus and calicivirus.

\section{DISCUSSION}

In the present study, the positivity rates for astrovirus and adenovirus were $3.1 \%$ and $3.6 \%$, respectively; these detection indices were similar to those previously found in the Central-West region of Brazil (Cardoso et al. 2002, Santos et al. 2007). However, other authors have reported higher detection rates in Brazil (Cardoso et al. 2002, Victoria et al. 2007, Resque et al. 2007). The highest detection rates were observed in epidemic situations (Silva et al. 2001) and, according to Resque et al. (2007), this could reflect socio-economic differences. Globally, detection rates vary from $1.8 \%-13.9 \%$, depending on the geographical area, as well the socio-economic situation (Jarecki-Khan et al. 1993, Caracciolo et al. 2007, Papaventsis et al. 2008).

The viruses belonging to the Norovirus and Sapovirus genera of the Caliciviridae family infect humans of all ages. They have been detected globally with indexes that vary from 7.3\%-23.7\% (Marrie-Cardine et al. 2002, Caracciolo et al. 2007). In Brazil, similar to astrovirus and adenovirus, there are few calicivirus detection studies and one of these showed detection rates of $14.5 \%$ in Rio de Janeiro (Soares et al. 2007). In the Central-West region, only one study was conducted, which showed a detection index of $8.6 \%$ (Borges et al. 2006), similar to that observed in the present study of $7.6 \%$.

In the present work, calicivirus samples were not characterized in terms of calicivirus genera. However, 22 were detected by Ni/E3 or JV12/13 primers and one was detected by Ni/E3 and 289/290 primers. Considering that the first two primer sets were designed to detect norovirus, our results suggest that these samples may belong to this genus. On the other hand, the 289/290 primer pair detects both norovirus and sapovirus, and rotavirus by cross-reactivity (Ludert et al. 2004). In this study, we found eight samples that were positive by this primer set. We did not use additional primers to confirm the presence of calicivirus; therefore it is not possible to conclude to which genera the samples belong. Additional studies utilizing DNA sequencing are in progress in our lab and should address this question.

The detection of astrovirus, adenovirus and calicivirus reveals that infection by these agents is a reality among our infantile population and suggests the possibility of nosocomial transmission, which has been previously observed for rotaviruses (Andreasi et al. 2007). During this study we were not informed about nosocomial outbreaks, however. This is the first study conducted in our state detecting enteric viruses that play a role in the etiology of childhood acute gastroenteritis.

Among the 85 children reporting respiratory complaints, no samples were positive for astrovirus. Conversely, three and seven children with respiratory symp- toms showed positivity to adenovirus and calicivirus, respectively. Unfortunately, in the present study, serotype identification, which could possibly suggest a cause for the respiratory symptoms, was not performed. These data are in agreement with a study that detected adenovirus in children with acute gastroenteritis and respiratory symptoms (Fodha et al. 2007).

Most of the co-infection cases observed in this study involved the association of one of the three other viruses with group A rotavirus, with one sample presenting an association between astrovirus and calicivirus. The existence of co-infections between gastroenteric viruses makes the determination of the primary etiologic agent, as well as the determination of virus-specific symptoms, difficult (Resque et al. 2007, Victória et al. 2007).

Astrovirus detection occurred mainly in the first day of diarrhea, a finding similar to the results reported by Gabbay et al. (2007). For adenovirus, the highest detection occurred in the third day, and for calicivirus, the highest positivity rates were observed on or after the fifth day of disease. Astrovirus and adenovirus were detected in similar proportions in children of both genders, while calicivirus predominated in female children. This finding is in agreement with published literature (Santos et al. 2007), although Shimizu et al. (2007) showed a higher detection of adenovirus among male children, and Borges et al. (2006) found no significant differences between genders regarding calicivirus infection.

Regarding the year of sample collection, we observed a higher positivity during the years of 2002 and 2003, with a predominance of calicivirus seen in 2002. This observation should be interpreted carefully, however, because, in the years 2000, 2001 and 2004, the number of samples was lower than the number collected in 2002 and 2003. The three viruses investigated were found to circulate in all months of the year, with no evident detection peak. Furthermore, despite the high occurrence of calicivirus infection in March and April, no statistically significant differences existed as compared to calicivirus infection found in other months of the year. This result is in accordance with those reported previously (Resque et al. 2007).

The results obtained from this study reinforce the need for the establishment of regional vigilance systems to evaluate the impact of enteric viruses on the etiology of viral acute gastroenteritis, especially considering the recent inclusion of the rotavirus vaccine in the childhood immunization calendar.

\section{REFERENCES}

Andreasi MSA, Batista SMF, Tozetti IA, Ozaki CO, Nogueira MM, Fiaccadori FS, Borges AMT, Santos RAT, Cardoso DDP 2007. Rotavirus A among hospitalized infants, up to three years of age, with acute gastroenteritis in Campo Grande, state of Mato Grosso do Sul. Rev Soc Bras Med Trop 40: 411-414.

Boom R, Sol CJA, Salimans MMM, Jansen CL, Wertheim-van Dillen PM E, van der Noordaa J 1990. Rapid and simple method for purification of nucleic acids. J Clin Microbiol 28: 495-503.

Borges AMT, Teixeira JMS, Costa PSS, Giugliano LG, Fiaccadori FS, Franco RC, Brito WMED, Leite JPG, Cardoso DDP 2006. Detection of calicivirus from fecal samples from children with 
acute gastroenteritis in the West Central region of Brazil. Mem Inst Oswaldo Cruz 101: 721-724.

Caracciolo S, Minini C, Colombrita D, Foresti I, Avolio M, Tosti G, Fiorentini S, Caruso A 2007. Detection of sporadic cases of Norovirus infection in hospitalized children in Italy. New Microbiol 30: $49-52$.

Cardoso DDP, Fiaccadori FS, Souza MBLD, Martins RMB, Leite JPG 2002. Detection and genotyping of astroviruses from children with acute gastroenteritis from Goiania, Goiás, Brazil. Med Sci Monit 8: 624-628.

Fodha I, Chouikha A, Dewar J, Trabelsi A, Boujaafar N, Steele AD 2007. Prevalence of adenovirus antigens in children presenting with acute diarrhoea. Med Trop 67: 256-258.

Gabbay YB, Linhares AC, Oliveira DS, Nakamura LS, Mascarenhas JDP, Gusmão RHP, Heinemann MB, Macedo O, Leite JPG 2007. First detection of a human astrovirus type 8 in a child with diarrhea in Belem, Brazil: comparison with other strains worldwide and identification of possible three lineages. Mem Inst Oswaldo Cruz 102: 531-534.

Girard MP, Steele D, Chaignat CL, Kieny MP 2006. A review of vaccine research and development: human enteric infections. Vaccine 24: 2732-2750.

Green J, Gallimore CI, Norcott JP, Lewis D, Brown DW 1995. Broadly reactive reverse transcriptase polymerase chain reaction for the diagnosis of SRSV-associated gastroenteritis. $J$ Med Virol 47: 392-398.

Jakab F, Peterfai J, Meleg E, Banyai K, Mitchell DK, Szucs G 2005. Comparison of clinical characteristics between astrovirus and rotavirus infections diagnosed in 1997 to 2002 in Hungary. Acta Paediatr 94: 667-671.

Jarecki-Khan K, Tzipori SR, Unicomb LE 1993. Enteric adenovirus infection among infants with diarrhea in rural Bangladesh. JClin Microbiol 31: 484-489.

Jiang X, Espul C, Zhong WM, Cuello H, Matson DO 1999. Characterization of a novel human calicivirus that may be a naturally occurring recombinant. Arch Virol 144: 2377-2387.

Ludert JE, Alcalá AC, Liprandi F 2004. Prime pair p289-p290, designed to detect both noroviruses and sapoviruses by reverse transcription-PCR, also detects rotaviruses by cross-reactivity. $J$ Clin Microbiol 42: 835-836.
Marie-Cardine A, Gourlain K, Mouterde O, Castignolles N, Hellot MF, Mallet E, Buffet-Janvresse C 2002. Epidemiology of acute viral gastroenteritis in children hospitalized in Rouen, France. Clin Infect Dis 34: 1170-1178.

Noel JS, Lee TW, Kurtz JB, Glass RI, Monroe SS 1995. Typing of human astroviruses from clinical isolates by enzyme immunoassay and nucleotide sequencing. J Clin Microbiol 33: 797-801.

Papaventsis DC, Dove W, Cunliffe NA, Nakagomi O, Combe P, Grosjean P, Hart CA 2008. Human astrovirus gastroenteritis in children, Madagascar, 2004-2005. Emerg Infect Dis 14: 844-846.

Pereira HG, Azeredo RS, Leite JP, Andrade ZP, De Castro L 1985. A combined enzyme immunoassay for rotavirus and adenovirus (EIARA). J Virol Methods 10: 21-28.

Resque HR, Munford V, Castilho JG, Schmich H, Caruzo TAR, Racz ML 2007. Molecular characterization of astrovirus in stool samples from children in Sao Paulo, Brazil. Mem Inst Oswaldo Cruz 102: 969-974.

Santos RAT, Borges AMT, Costa PSS, Teixeira JMS, Giugliano LG, Leite JPG, Cardoso DDP 2007. Astrovirus infection in children living in the Central West region of Brazil. Mem Inst Oswaldo Cruz 102: 209-213.

Shimizu H, Phan TG, Nishimura S, Okitsu S, Maneekarn N, Ushijima H 2007. An outbreak of adenovirus serotype 41 infection in infants and children with acute gastroenteritis in Maizuru City, Japan. Infect Genet Evol 7: 279-284.

Silva AMV, Leite EG, Assis RMS, Majerowicz S, Leite JPG 2001. An outbreak of gastroenteritis associated with astrovirus serotype 1 in a day care center, in Rio de Janeiro, Brazil. Mem Inst Oswaldo Cruz 96: 1069-1073.

Soares CC, Santos N, Beard RS, Albuquerque MC, Maranhão AG, Rocha LN, Ramirez ML, Monroe SS, Glass RI, Gentsch J 2007. Norovirus detection and genotyping for children with gastroenteritis, Brazil. Emerg Infect Dis 13: 1244-1246.

Victoria M, Carvalho-Costa FA, Heinemann MB, Leite JPG, Miagostovich MP 2007. Genotypes and molecular epidemiology of human astroviruses in hospitalized children with acute gastroenteritis in Rio de Janeiro, Brazil. J Med Virol 79: 939-944.

Vinjé J, Altena SA, Koopmans MP 1997. The incidence and genetic variability of small round-structured viruses in outbreaks of gastroenteritis in The Netherlands. J Infect Dis 176: 1374-1378.

Wilhelmi I, Roman E, Sanchez-Fauquier A 2003. Viruses causing gastroenteritis. Clin Microbiol Infect 9: 247-262. 